\title{
Aging exacerbates development of cerebral microbleeds in a mouse model
}

\author{
Rachita K. Sumbria ${ }^{1,2}$, Mher Mahoney Grigoryan², Vitaly Vasilevko³, Annlia Paganini-Hill², Kelley Kilday3, \\ Ronald Kim ${ }^{4}$, David H. Cribbs ${ }^{3}$ and Mark J. Fisher ${ }^{2,4,5,6^{*}}$
}

\begin{abstract}
Background: Cerebral microhemorrhages $(\mathrm{CMH})$ are commonly found in the aging brain. $\mathrm{CMH}$ are also the neuropathological substrate of cerebral microbleeds (CMB), demonstrated on brain MRI. Recent studies demonstrate the importance of systemic inflammation in $\mathrm{CMH}$ development, but the relationships among inflammation, aging, and $\mathrm{CMH}$ development are not well-defined. In the current study, we hypothesized that the pathogenesis of inflammation-induced $\mathrm{CMH}$ in mice differs by age.

Methods: We studied young (3 months, $n=20)$ and old (18 months, $n=25)$ C57BL/6 mice injected with low-dose lipopolysaccharide (LPS; 1 mg/kg, i.p.) or saline at 0, 6, and $24 \mathrm{~h}$. Seven days after the first LPS/saline injection, brains were harvested, sectioned, and stained with hematoxylin and eosin (H\&E) and Prussian blue (PB) to estimate acute/fresh and sub-acute $\mathrm{CMH}$ development, respectively. The relationships between microglial/macrophage activation (ionized calcium-binding adapter molecule-1), astrocyte activation (glial fibrillary acidic protein), blood-brain barrier (BBB) disruption (brain immunoglobulin G), aging, and $\mathrm{CMH}$ development were examined using immunohistochemistry.
\end{abstract}

Results: Aging alone did not increase spontaneous H\&E-positive CMH development but significantly increased the number, size, and total area of LPS-induced H\&E-positive CMH in mice. LPS- and saline-treated aged mice had significantly larger PB-positive CMH compared with young mice, but the total area of PB-positive CMH was increased only in LPS-treated aged mice. Aged mice had significantly increased microglial/macrophage activation, which correlated with H\&E- and PB-positive CMH development. Aged mice treated with LPS had significantly increased astrocyte activation and BBB disruption compared with young LPS-treated mice.

Conclusions: Aging makes the brain more susceptible to inflammation-induced $\mathrm{CMH}$ in mice, and this increase in $\mathrm{CMH}$ with aging is associated with microglial/macrophage activation.

Keywords: Animal models, Cerebral microhemorrhage, Cerebral microbleeds, Inflammation, Hemosiderin, Aging

\section{Background}

Age is the most significant independent risk factor for cerebral microbleeds $(\mathrm{CMB})$, which are identified by brain MRI and have as their pathologic substrate cerebral microhemorrhages $(\mathrm{CMH})[1,2]$. In addition to aging, $\mathrm{CMB}$ are associated with hypertension, cerebral amyloid angiopathy (CAA), Alzheimer's disease, cerebral autosomal-dominant arteriopathy with subcortical infarcts and leukoencephalopathy (CADASIL), and chronic

\footnotetext{
* Correspondence: mfisher@uci.edu

2Department of Neurology, University of California, Irvine, CA, USA

${ }^{4}$ Department of Pathology and Laboratory Medicine, University of California, Irvine, CA, USA

Full list of author information is available at the end of the article
}

kidney disease, among other diseases [3-7]. Despite their high prevalence and clinical importance, the mechanisms underlying age-related $\mathrm{CMB}$ increase are not wellunderstood. One mechanism suggested by clinical findings is the activation of inflammatory cascades followed by blood-brain barrier (BBB) damage resulting in $\mathrm{CMH}$ development [8-11]. In fact, a pro-inflammatory systemic state is a common factor observed in normal aging and diseases with a high prevalence of CMB [12-15].

Recently, we showed that systemic inflammation induced by lipopolysaccharide (LPS) administration results in $\mathrm{CMH}$ development in mice and that $\mathrm{CMH}$ development was associated with levels of systemic 
and neuroinflammatory markers $[2,16]$. However, the relationships among aging, inflammation, and $\mathrm{CMH}$ development are not well-defined [3]. The current study aimed to determine if aging exacerbates inflammationinduced $\mathrm{CMH}$, and we hypothesized that the pathogenesis of inflammation-induced $\mathrm{CMH}$ in mice differs by age. We utilized the inflammation-induced $\mathrm{CMH}$ mouse model which results in MRI-demonstrable CMB [2]. LPS was used as the inflammatory stimulus to induce $\mathrm{CMH}$ development in young (3-month old) and aged (18-month old) mice. Age-related changes in both hematoxylin and eosin (H\&E)-positive (acute) and Prussian blue-positive (subacute) $\mathrm{CMH}$ were examined in relationship to markers of neuroinflammation and BBB damage.

\section{Methods}

\section{Mouse treatment}

All animal procedures were approved by the University of California, Irvine, Institutional Animal Care and Use Committee, and followed the ARRIVE Guidelines for animal experiments reporting. Young (3-month old) and aged (18-month old) C57BL/6 male and female mice (National Institute of Aging, Bethesda, MD) were randomly assigned to two treatment groups: one treated with a $1 \mathrm{mg} / \mathrm{kg}$ dose of LPS derived from gram-negative bacterium Salmonella typhimurium (Sigma-Aldrich, St. Louis, MO) and the other treated with saline intraperitoneally (i.p.) at three times at 0,6 , and $24 \mathrm{~h}$. Seven days after the first LPS/saline injection, mice were anesthetized with a lethal dose of Nembutal (150 mg/kg, i.p.), cardiac perfusions were performed using ice-cold PBS, and brains were processed for $\mathrm{CMH}$ detection as described in our previous work [16]. The sample size for each group was as follows: young-saline $=10$, agedsaline $=10$, young-LPS $=10$, and aged-LPS $=15$. The average weight of the mice was $28.1 \pm 0.9 \mathrm{~g}$ at the beginning of the study and $27.1 \pm 0.8$ at the end of the study.

\section{Microhemorrhage detection}

Right hemispheres were fixed in $4 \%$ paraformaldehyde (Boston BioProducts, Ashland, MA) at $4{ }^{\circ} \mathrm{C}$, examined for surface $\mathrm{CMH}$, and sectioned into $40-\mu \mathrm{m}$ coronal sections using a vibratome (Technical Products International, Inc., St. Louis, MO). Every fourth, fifth, sixth, and seventh section was collected. Every sixth section was stained with H\&E by Research Services Core offered by the Department of Pathology and Laboratory Medicine at UCI Medical Center to detect fresh (acute) $\mathrm{CMH}$. Every seventh section was stained with Prussian blue $(\mathrm{PB})$ to detect hemosiderin (a marker of sub-acute $\mathrm{CMH}$ ) [16]. Briefly, sections were stained using 5\% potassium hexacyanoferrate trihydrate (Sigma, St. Louis, MO) and 5\% hydrochloric acid (Sigma, St. Louis, MO) for $30 \mathrm{~min}$, rinsed in water and counterstained with
Nuclear Fast Red (Sigma, St. Louis, MO), dehydrated, and cover slipped. Remaining sections were used for immunohistochemistry. $\mathrm{CMH}$ were counted at a $\times 20 \mathrm{mag}$ nification by a blinded observer, and digitized images were used to determine $\mathrm{CMH}$ size $\left(\mu \mathrm{m}^{2}\right)$ and positive area (expressed as a percent of total area analyzed), by an observer blinded to the experiment using RGB CMH analyzer program and $\mathrm{NIH}$ Image $\mathrm{J}$ software 1.62, respectively [16].

\section{IgG, Iba-1, and GFAP immunohistochemistry}

Parenchymal IgG (BBB damage marker), Iba-1 (microglial/macrophage marker), and GFAP (astrocyte marker) immunohistochemistry were performed [16]. Briefly, 40$\mu \mathrm{m}$ sections were incubated in $0.5 \%$ hydrogen peroxide in $0.1 \mathrm{M}$ PBS (pH 7.4) containing 0.3\% Triton-X100 (phosphate-buffered saline with triton-X100 (PBST)) for $30 \mathrm{~min}$ at room temperature (RT), washed with PBST, and blocked with PBST containing $2 \%$ bovine serum albumin for $30 \mathrm{~min}$ at RT. Sections were then incubated overnight at $4{ }^{\circ} \mathrm{C}$ with a rabbit anti-mouse IgG antibody (1:200 dilution; Jackson ImmunoResearch, West Grove, PA), rabbit antibody against Iba-1 (1:200 dilution, Wako Chemicals USA, Richmond, VA), or rabbit antibody against GFAP (1:2000 dilution, Abcam, Cambridge, MA). After washing with PBST, sections were incubated at RT for $1 \mathrm{~h}$ with biotinylated anti-rabbit IgG (1:500 dilution; Jackson ImmunoResearch, West Grove, PA), followed by $1 \mathrm{~h}$ incubation at RT with $\mathrm{ABC}$ complex, and developed with 3,3'-diaminobenzidine (DAB) (Vector Laboratories, Burlingame, CA) as per manufacturer's instructions. Sixteen images per brain section were acquired at $\times 20$ magnification, and the total positive immunoreactive area (expressed as percent of total analyzed area) was quantified using NIH Image J software 1.62 by an observer blinded to the experimental groups [16].

\section{Statistical analysis}

Based on our previous work showing a mean number of $\mathrm{CMH}$ per brain section of $1.3 \pm 0.8$ in the LPS group and $0.03 \pm 0.05$ in the saline group, we estimated that at least 10 mice per group were needed to detect a significant difference between LPS and control mice at $80 \%$ power. One-way ANOVA with Bonferroni's post hoc test for normal data and Kruskal-Wallis with Dunn's multiple comparison test for non-normal data were used to compare more than two groups. Two-way ANOVA with Tukey's post hoc test was used for sex comparisons. Pearson $r$ correlation was used for correlation analysis. One-sample $t$ test was used to compare group means with a hypothesized mean $=0$ when the values in the control group were zero (e.g., surface microhemorrhages). A two-tailed $p$ value of $<0.05$ was considered statistically significant. 


\section{Results}

\section{Survival}

All the saline-treated young and aged mice and LPS-treated young mice survived the duration of the study $(n=10$ per group). Four aged mice treated with LPS died prematurely reducing the number included in the analysis to 15 .

\section{Cerebral microhemorrhages}

Aging exacerbated the development of LPS-induced surface $\mathrm{CMH}$, and the number of surface $\mathrm{CMH}$ in LPStreated aged mice increased 23-fold compared with LPStreated young mice (Fig. 1a, e). No surface $\mathrm{CMH}$ were observed in saline-treated mice, young or old.

In young mice, no spontaneous (in the absence of LPS) H\&E-positive acute CMH were observed in salinetreated mice and a $1 \mathrm{mg} / \mathrm{kg}$ triple dose of LPS did not result in significant development of H\&E-positive acute $\mathrm{CMH}$ (Fig. 1b-d, f). Aging did not cause a significant increase in spontaneous acute $\mathrm{CMH}$ development in saline-treated mice; however, similar to the effect on surface $\mathrm{CMH}$ development, aging significantly increased the development of H\&E-positive acute parenchymal $\mathrm{CMH}$ in LPS-treated mice (Fig. 1b-d, f). All three parameters (number, size, and total area) of H\&E-positive acute parenchymal $\mathrm{CMH}$ were significantly increased with age in LPS-treated mice (Fig. 1b-d). H\&E-positive lesions were significantly higher in the cerebellum compared with the cortex and the sub-cortex (data not shown).

The number of PB-positive sub-acute parenchymal $\mathrm{CMH}$ was significantly higher in the LPS-treated young and LPS-treated aged mice compared with their respective saline controls (Fig. $2 \mathrm{a}-\mathrm{c}$ ). The number of PBpositive $\mathrm{CMH}$ lesions increased twofold in LPS-treated aged mice compared with LPS-treated young mice, although this increase did not reach statistical significance. Aging independently (with and without LPS) caused a significant increase in PB-positive lesion size; average size increased approximately threefold in both saline- and LPS-treated aged mice compared with their respective young controls (Fig. 2b). Total PB-positive lesion area, which is a function of both lesion number and size, was significantly increased with age in LPStreated mice (Fig. 2c). The number of H\&E-positive acute and PB-positive sub-acute lesions were significantly correlated (Pearson $r=0.92, p<0.0001$ ) in aged mice (Fig. 2d) but not in young mice (data not shown).

\section{Relationship between CMH development, neuroinflammation, BBB damage, and aging}

Aging was associated with a significant increase in brain Iba-1-positive immunoreactivity, independent of LPS treatment (Fig. 3a). Immunoreactive area of the

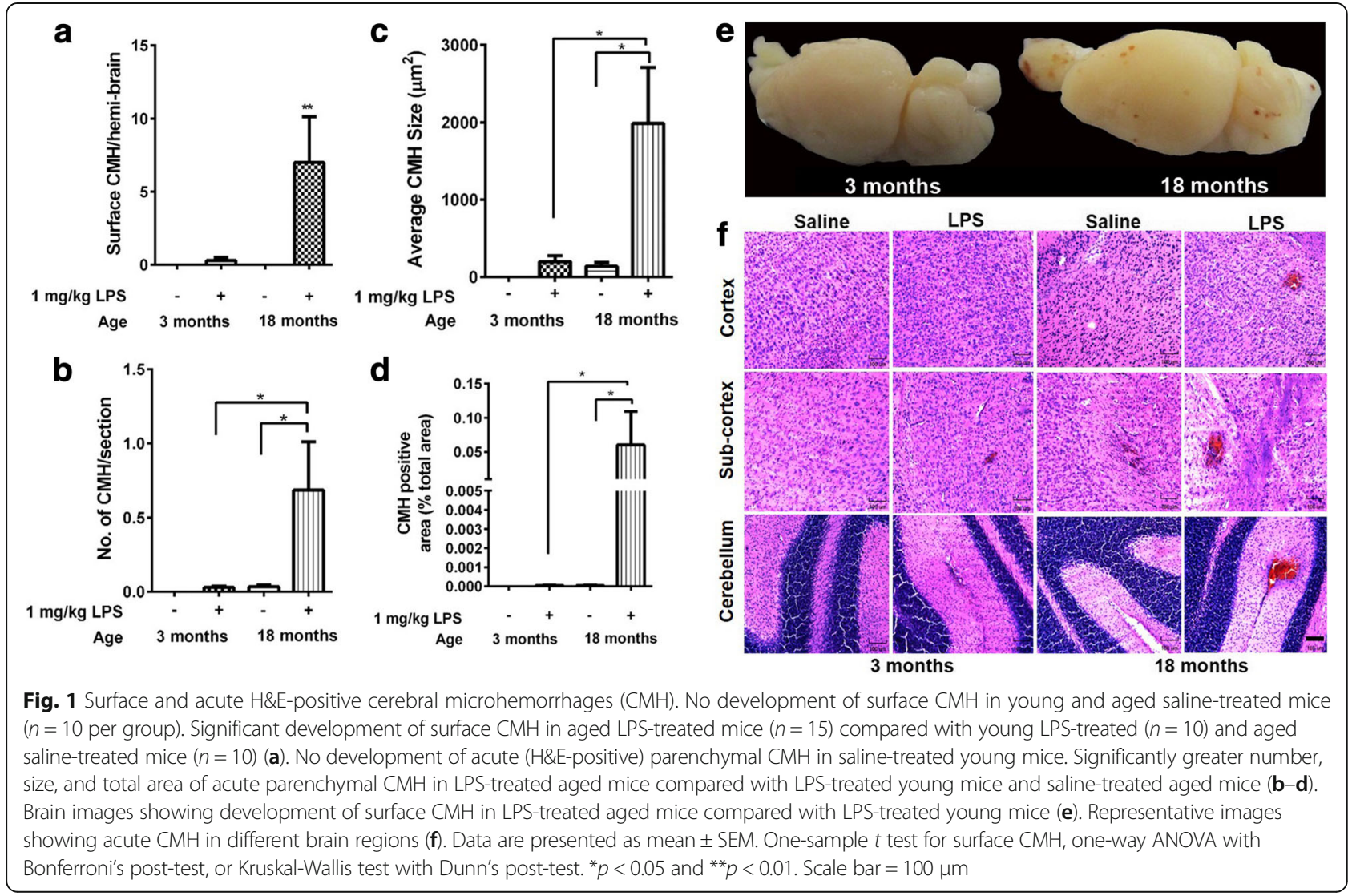



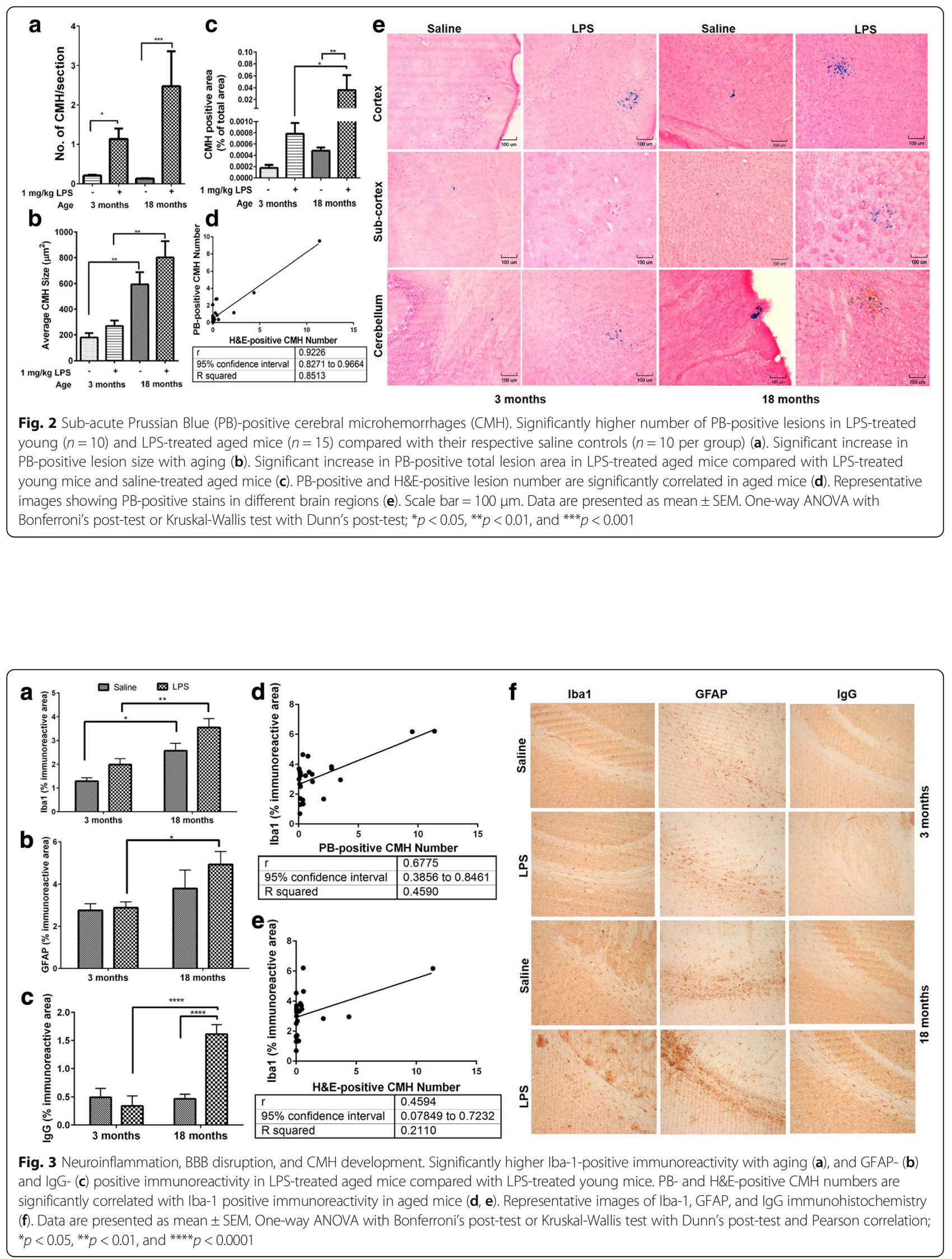
astrocyte activation marker (GFAP) and BBB damage marker (brain $\operatorname{IgG}$ ) on the other hand were significantly increased with age only in LPS-treated mice (Fig. 3b, c). Overall, both the H\&E-positive and PB-positive lesion numbers were significantly correlated with Iba-1-positive immunoreactivity (Fig. 3d, e).

\section{Sex and CMH development}

In an exploratory analysis of the relationship between sex and $\mathrm{CMH}$ development, the number $(p<0.01)$, size $(p<0.0001)$, and total area $(p<0.01)$ of H\&E-positive acute parenchymal CMH were increased with age in LPStreated male mice compared with LPS-treated female aged mice. No sex differences were observed in PB-positive $\mathrm{CMH}$ and in markers of neuroinflammation and BBB injury among young or aged mice (data not shown).

\section{Discussion}

In the current study, we investigated the effect of aging on inflammation-induced $\mathrm{CMH}$ development in a mouse model. We demonstrated negligible spontaneous acute $\mathrm{CMH}$ development in saline-treated young and aged mice. Exacerbation of acute $\mathrm{CMH}$ development was observed in LPS-treated aged mice and not in LPS-treated young mice. Since H\&E staining enables the detection of fresh or acute microhemorrhages in the brain [2], the presence of fresh microhemorrhages in aged LPS-treated mice suggests that inflammation-induced CMH development is an ongoing process that lasts days after the last LPS injection in aged mice but not young mice (last LPS injection at day 2 and sacrifice at day 7 in the current study). PB on the other hand detects hemosiderin that remains at the bleeding site for a prolonged period of time, thus enabling the detection of cumulative $\mathrm{CMH}$ load at the time of sacrifice.

LPS treatment resulted in significant development of PB-positive sub-acute $\mathrm{CMH}$ in both young and aged mice. Consistent with other findings and our own work, we found low numbers of spontaneous PB-positive $\mathrm{CMH}$ in young and aged mice $[2,17]$. Total PB-positive $\mathrm{CMH}$ load was significantly higher in LPS-treated aged mice and not in LPS-treated young mice. Spontaneous PB-positive CMH were significantly larger in aged mice, but this effect was independent of LPS treatment. The number of PB-positive lesions was highly associated with the number of H\&E-positive lesions $\left(r^{2}=0.85\right)$ further suggesting that hemosiderin-positive sub-acute microhemorrhages detected by PB may help predict the susceptibility of the aging brain to develop acute or fresh microhemorrhages. Taken together, these results show that aging and inflammation together make the brain more vulnerable to $\mathrm{CMH}$ development and further corroborate previous studies that support the role of inflammation in the pathogenesis of $\mathrm{CMH}[8,9,18]$.
Though the exact molecular mechanisms underlying inflammation-induced $\mathrm{CMH}$ development are not completely understood, loss of microvascular integrity associated with inflammatory changes is associated with $\mathrm{CMH}$ development [19]. In the current study, we observed an increase in BBB disruption in LPS-treated aged mice consistent with our previous work [16]. Systemic administration of LPS is known to result in BBB injury and inflammation by altering tight junction protein expression, enlarging intercellular clefts, and increasing cytokine and chemokine release [20,21]. These effects of LPS are mediated by its binding to toll-like receptor 4 (TLR4), which activates signaling pathways implicated in BBB disruption [22]. The contribution of TLR4 in intracerebral hemorrhage (ICH)-induced brain injury and inflammation has been documented [23], and a recent study showed that endothelial TLR4 activation by its canonical ligand LPS accelerates cerebral cavernous malformations (CCM) [24]. Given the role of TLR4 activation in $\mathrm{ICH}$ and $\mathrm{CCM}$ development and increased expression of TLR4 with the LPS dose used in the current study [25], TLR4 activation via LPS may mediate at least some of the effects observed in the current study. Overall, increased LPS-mediated BBB disruption in the current study further supports the role of the inflammation-induced loss of BBB integrity in $\mathrm{CMH}$ development [11]; the underlying role of TLR4 in $\mathrm{CMH}$ development needs further investigation. Loss of BBB integrity detected at the time of sacrifice in the aged mice in the current study may further explain the presence of acute CMH several days after the last LPS injection in aged mice and not LPS-treated young mice.

Studies show that hemosiderin deposits in the brain are often surrounded by macrophages which can further initiate an inflammatory response [26]. Consistent with this and our previous work, we found a significant increase in two different markers of neuroinflammation, Iba-1 (microglial/macrophage activation marker) and GFAP (astrocyte activation marker), in aged mice [2]. Iba-1 immunoreactivity was elevated with aging in the current study and was independent of LPS treatment. Further, we found that microglia/macrophage activation was significantly associated with $\mathrm{CMH}$ development in aged mice. We also found an increase in astrocyte activation in the aged mice treated with LPS, further indicating the increased susceptibility of the aging brain to neuroinflammation.

The role of microglia/macrophages in the pathogenesis of $\mathrm{CMH}$ is not well-defined. Apart from exhibiting a cytotoxic phenotype (M1 phenotype), microglia/macrophages can also mediate repair mechanisms in the brain depending on the brain microenvironment (M2 phenotype) [27, 28]. M2 macrophages are associated with hematoma resolution after intracerebral hemorrhage in a 
mouse model [29]. Whether the macrophage/microglial response to $\mathrm{CMH}$ observed in the current study is protective (clearing the $\mathrm{CMH}$ ) or detrimental (triggering local inflammation) needs further investigation.

Sex differences in CMB development have been reported in clinical settings and $\mathrm{CMB}$ are more prevalent in males [30]. An exploratory analysis of our data showed that LPS-treated aged male mice were more susceptible to acute (H\&E-positive) $\mathrm{CMH}$ development compared to LPS-treated aged female mice. We observed no sex differences in subacute (PB-positive) $\mathrm{CMH}$ development in aged mice. These sex differences in LPS-induced CMH development are consistent with prior observations on sex differences in response to LPS [31]. We speculate that inflammation-induced $\mathrm{CMH}$ development is an ongoing process in aged male mice at the time of sacrifice (7 days), compared with aged female mice. Future experimental studies are needed to fully define this process.

\section{Conclusions}

The current study highlights the relationships among aging, inflammation, and CMH development. Aging increases the susceptibility of the brain to inflammationinduced $\mathrm{CMH}$ development in mice, and this increase in $\mathrm{CMH}$ development is significantly associated with microglial/macrophage activation. The exact role of microglial activation in $\mathrm{CMH}$ pathogenesis needs further investigation.

\section{Abbreviations}

BBB: Blood-brain barrier; CAA: Cerebral amyloid angiopathy;

CADASIL: Cerebral autosomal-dominant arteriopathy with subcortical infarcts and leukoencephalopathy; CCM: Cerebral cavernous malformations;

CMB: Cerebral microbleeds; CMH: Cerebral microhemorrhages; DAB: 3,3'Diaminobenzidine; GFAP: Glial fibrillary acidic protein; H\&E: Hematoxylin and eosin; Iba-1: Ionized calcium-binding adapter molecule-1; ICH: Intracerebral hemorrhage; IgG: Immunoglobulin G; LPS: Lipopolysaccharide; PB: Prussian blue

\section{Acknowledgements}

We would like to thank Tuan Ngo, Gurjit Pannu, Jiwei Cheng, and Masood Akram for their assistance.

\section{Funding}

Funding for this study was provided by a grant from NINDS: RO1 NS20989 (MJF and DHC).

\section{Availability of data and materials}

Community-established norms of data sharing are fully respected.

\footnotetext{
Authors' contributions

RKS designed and performed experiments, analyzed data, prepared the figures, and wrote the paper. MMG performed experiments and collected data. VV and KK performed the Prussian blue and immunohistochemical staining. APH helped with data analysis. RK provided expertise with mouse brain histology. DHC participated in the conception of the study and was involved in revising the manuscript critically for important intellectual content. MJF conceived the study, designed and coordinated the experiments, and helped in the drafting and editing of the manuscript. All authors read and approved the final manuscript.
}

\section{Ethics approval}

All animal procedures were approved by UCl Institutional Animal Care and Use Committee and were carried out in compliance with University Laboratory Animal Resources regulations. This work does not involve any applicable consent to participate.

\section{Consent for publication \\ Not applicable}

\section{Competing interests}

Mark J. Fisher has received support from Boehringer-Ingelheim and Otsuka Pharmaceutical Company (research grants).

\section{Publisher's Note}

Springer Nature remains neutral with regard to jurisdictional claims in published maps and institutional affiliations.

\section{Author details}

${ }^{1}$ Department of Biopharmaceutical Sciences, School of Pharmacy, Keck Graduate Institute, Claremont, CA, USA. ${ }^{2}$ Department of Neurology, University of California, Irvine, CA, USA. IInstitute for Memory Impairments and Neurological Disorders, University of California, Irvine, CA, USA. ${ }^{4}$ Department of Pathology and Laboratory Medicine, University of California, Irvine, CA, USA. ${ }^{5}$ Department of Anatomy and Neurobiology, University of California, Irvine, CA, USA. ${ }^{6}$ UC Irvine Medical Center, 101 The City Drive South, Shanbrom Hall, Room 121, Orange, CA 92868, USA.

Received: 21 November 2017 Accepted: 7 February 2018

Published online: 06 March 2018

\section{References}

1. Greenberg SM, Vernooij MW, Cordonnier C, Viswanathan A, Al-Shahi Salman $\mathrm{R}$, Warach $\mathrm{S}$, et al. Cerebral microbleeds: a guide to detection and interpretation. Lancet Neurol. 2009;8(2):165-74.

2. Sumbria RK, Grigoryan MM, Vasilevko V, Krasieva TB, Scadeng M, Dvornikova AK, et al. A murine model of inflammation-induced cerebral microbleeds. J Neuroinflammation. 2016;13(1):218.

3. Ungvari Z, Tarantini S, Kirkpatrick AC, Csiszar A, Prodan Cl. Cerebral microhemorrhages: mechanisms, consequences, and prevention. Am J Physiol Heart Circ Physiol. 2017;312(6):H1128-H43.

4. Li L, Fisher M, Lau WL, Moradi H, Cheung A, Thai G, et al. Cerebral microbleeds and cognitive decline in a hemodialysis patient: case report and review of literature. Hemodial Int. 2015;19(3):E1-7.

5. Lee JS, Ko K, Oh JH, Park JH, Lee HK, Floriolli D, et al. Cerebral microbleeds, hypertension, and intracerebral hemorrhage in cerebral autosomaldominant arteriopathy with subcortical infarcts and leukoencephalopathy. Front Neurol. 2017;8:203

6. Cordonnier $C$, van der Flier WM. Brain microbleeds and Alzheimer's disease: innocent observation or key player? Brain. 2011;134(Pt 2):335-44.

7. Vernooij MW, van der Lugt A, Ikram MA, Wielopolski PA, Niessen WJ, Hofman A, et al. Prevalence and risk factors of cerebral microbleeds: the Rotterdam Scan Study. Neurology. 2008;70(14):1208-14.

8. Miwa K, Tanaka M, Okazaki S, Furukado S, Sakaguchi M, Kitagawa K. Relations of blood inflammatory marker levels with cerebral microbleeds. Stroke. 2011:42(11):3202-6.

9. Shoamanesh A, Preis SR, Beiser AS, Vasan RS, Benjamin EJ, Kase CS, et al. Inflammatory biomarkers, cerebral microbleeds, and small vessel disease: Framingham Heart Study. Neurology. 2015;84(8):825-32.

10. Schreiber S, Bueche CZ, Garz C, Braun H. Blood brain barrier breakdown as the starting point of cerebral small vessel disease? - new insights from a rat model. Exp Transl Stroke Med. 2013;5(1):4.

11. van Nieuwenhuizen KM, Hendrikse J, Klijn CJM. New microbleed after blood-brain barrier leakage in intracerebral haemorrhage. BMJ Case Rep. 2017. https://doi.org/10.1136/bcr-2016-218794

12. Dziedzic T. Systemic inflammation as a therapeutic target in acute ischemic stroke. Expert Rev Neurother. 2015;15(5):523-31.

13. Rouhl RP, Damoiseaux JG, Lodder J, Theunissen RO, Knottnerus IL, Staals J, et al. Vascular inflammation in cerebral small vessel disease. Neurobiol Aging. 2012;33(8):1800-6. 
14. Franceschi C, Campisi J. Chronic inflammation (inflammaging) and its potential contribution to age-associated diseases. J Gerontol A Biol Sci Med Sci. 2014;69(Suppl 1):S4-9.

15. Pauletto P, Rattazzi M. Inflammation and hypertension: the search for a link. Nephrol Dial Transplant. 2006;21(4):850-3.

16. Sumbria RK, Vasilevko V, Grigoryan MM, Paganini-Hill A, Kim R, Cribbs DH, et al. Effects of phosphodiesterase $3 \mathrm{~A}$ modulation on murine cerebral microhemorrhages. J Neuroinflammation. 2017;14(1):114.

17. Cacciottolo M, Morgan TE, Finch CE. Rust on the brain from microbleeds and its relevance to Alzheimer studies: invited commentary on Cacciottolo Neurobiology of Aging, 2016. J Alzheimers Dis Parkinsonism. 2016;6(6):287.

18. Romero JR, Preis SR, Beiser AS, DeCarli C, Lee DY, Viswanathan A, et al. Lipoprotein phospholipase A2 and cerebral microbleeds in the Framingham Heart Study. Stroke. 2012;43(11):3091-4.

19. Charidimou A, Werring DJ. A raging fire in acute lacunar stroke: inflammation, blood-brain barrier dysfunction and the origin of cerebral microbleeds. J Neurol Sci. 2014;340(1-2):1-2.

20. Banks WA, Gray AM, Erickson MA, Salameh TS, Damodarasamy M, Sheibani $\mathrm{N}$, et al. Lipopolysaccharide-induced blood-brain barrier disruption: roles of cyclooxygenase, oxidative stress, neuroinflammation, and elements of the neurovascular unit. J Neuroinflammation. 2015;12:223.

21. Ghosh A, Birngruber T, Sattler W, Kroath T, Ratzer M, Sinner F, et al. Assessment of blood-brain barrier function and the neuroinflammatory response in the rat brain by using cerebral open flow microperfusion (cOFM). PLoS One. 2014;9(5):e98143.

22. Nagyoszi P, Wilhelm I, Farkas AE, Fazakas C, Dung NT, Hasko J, et al. Expression and regulation of toll-like receptors in cerebral endothelial cells. Neurochem Int. 2010;57(5):556-64.

23. Fang H, Wang PF, Zhou Y, Wang YC, Yang QW. Toll-like receptor 4 signaling in intracerebral hemorrhage-induced inflammation and injury. J Neuroinflammation. 2013;10:27.

24. Tang AT, Choi JP, Kotzin JJ, Yang Y, Hong CC, Hobson N, et al. Endothelial TLR4 and the microbiome drive cerebral cavernous malformations. Nature. 2017;545(7654):305-10.

25. Chung DW, Yoo KY, Hwang IK, Kim DW, Chung JY, Lee CH, et al. Systemic administration of lipopolysaccharide induces cyclooxygenase-2 immunoreactivity in endothelium and increases microglia in the mouse hippocampus. Cell Mol Neurobiol. 2010;30(4):531-41.

26. Rosidi NL, Zhou J, Pattanaik S, Wang P, Jin W, Brophy M, et al. Cortical microhemorrhages cause local inflammation but do not trigger widespread dendrite degeneration. PLoS One. 2011;6(10):e26612.

27. Min H, Jang YH, Cho IH, Yu SW, Lee SJ. Alternatively activated braininfiltrating macrophages facilitate recovery from collagenase-induced intracerebral hemorrhage. Mol Brain. 2016;9:42.

28. Kigerl KA, Gensel JC, Ankeny DP, Alexander JK, Donnelly DJ, Popovich PG. Identification of two distinct macrophage subsets with divergent effects causing either neurotoxicity or regeneration in the injured mouse spinal cord. J Neurosci. 2009;29(43):13435-44.

29. Ni W, Mao S, Xi G, Keep RF, Hua Y. Role of erythrocyte CD47 in intracerebral hematoma clearance. Stroke. 2016:47(2):505-11.

30. Jeerakathil T, Wolf PA, Beiser A, Hald JK, Au R, Kase CS, et al. Cerebral microbleeds: prevalence and associations with cardiovascular risk factors in the Framingham Study. Stroke. 2004;35(8):1831-5.

31. Cai KC, van Mil S, Murray E, Mallet JF, Matar C, Ismail N. Age and sex differences in immune response following LPS treatment in mice. Brain Behav Immun. 2016;58:327-37.

\section{Submit your next manuscript to BioMed Central and we will help you at every step:}

- We accept pre-submission inquiries

- Our selector tool helps you to find the most relevant journal

- We provide round the clock customer support

- Convenient online submission

- Thorough peer review

- Inclusion in PubMed and all major indexing services

- Maximum visibility for your research

Submit your manuscript at www.biomedcentral.com/submit
Biomed Central 